\title{
Stem cells reveal mechanisms of myotonic dystrophy type 1
}

Experiments in mutant human embryonic stem cells (hESCs) have provided new insights into the neuropathological mechanisms underlying myotonic dystrophy type 1 (DM1), report Cécile Martinat and colleagues in Cell Stem Cell. "A genome-wide comparison of DM1 hESCs, differentiated into neural cells, with their healthy counterparts allowed the identification of reduced expression of two genes of the SLITRK family in mutant cells that was mirrored in DM1 brain samples," says Martinat, who is based at I-Stem, a French institute dedicated to the use of pluripotent stem cells to develop therapeutic strategies for monogenic diseases.

DM1 is the most common inherited form of adult-onset muscular dystrophy, and is caused by a CTG triplet repeat expansion in the 3'-untranslated region of the dystrophia myotonica-protein kinase (DMPK) gene. Previous research has indicated that this expansion causes $D M P K$ transcripts to accumulate in the nucleus, forming inclusions that sequester proteins involved in RNA processing. The DM1 expansion is, therefore, predicted to disrupt the expression of a variety of genes.

Martinat and her team used hESC lines derived from embryos in which the expanded $D M P K$ allele had been detected during preimplantation diagnosis. "Our goal was to evaluate whether DM1 hESCs could be used to unravel new
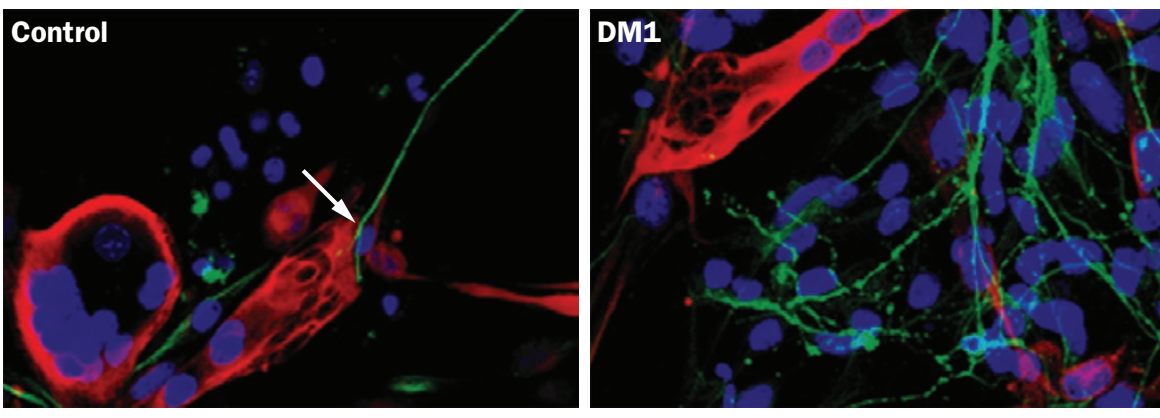

Arrow indicates interaction between healthy hESC-derived neurons (green) and human primary muscle cells (red), which is defective in DM1 neurons. Images provided by Dr Cécile Martinat.

cellular and molecular neuropathological mechanisms," she explains. Through whole-genome expression profiling and quantitative PCR, the researchers identified a number of genes that were differentially expressed between DM1 and wild-type hESCs after differentiation into neural precursor cells. Notably, the DM1 cells showed downregulation of SLITRK2 and SLITRK4, which encode proteins involved in neurite outgrowth and synaptogenesis.

In light of these findings, Martinat and colleagues examined the effects of the $D M P K$ expansion on synaptogenesis and neuritogenesis in hESCs differentiated into motor neurons. The DM1 hESCderived neurons showed increased neurite outgrowth compared with wild-type cells, but were substantially impaired in their ability to form neuromuscular connections when cocultured with human primary myotubes.

"These results illustrate the clinically important contribution of disease-specific hESCs to understanding human disease," says Marc Peschanski, Head of I-Stem. "This technology can help to unveil the changes in connectivity properties between neurons that are affected by a given neurological disease." The team now plans to use DM1 hESCs to screen for compounds that can rescue the mutant phenotype, which could lead to the development of new therapies for this currently untreatable disease.

Heather Wood

Original article Marteyn, A. et al. Mutant human embryonic stem cells reveal neurite and synapse formation defects in type 1 myotonic dystrophy. Cell Stem Cell 8,1-11 (2011) 